\section{Suchttherapie und kontrollierter Konsum von Opiaten und Kokain/Crack}

Zusammenfassung: Die Suchtmedizin erforscht die Sucht und deshalb eben gerade nicht diejenigen, die trotz Drogengebrauchs von der Sucht verschont bleiben. Eine intensivere Erforschung des kontrollierten Konsums von bekannten Suchtdrogen wie z. B. Heroin und Kokain stößt vor allem deshalb auf Widerstände, weil davon ein falsches Signal - eine Verharmlosung der Drogen und die Botschaft, dass man sie ruhig und ohne Angst vor dem Süchtigwerden konsumieren könne - befürchtet wird. Dieser Sichtweise ist jedoch nachdrücklich zu widersprechen. Zwar ehrt es die Suchtmedizin, dass es ihr um die Hilfsbedürftigen geht: um die schwangeren Konsumentinnen, die HIV-Infizierten, die schwerstkranken und völlig verelendeten Süchtigen. Gerade die Erforschung der Bedingungen, die Menschen dazu verhelfen, Suchtdrogen kontrolliert zu sich zu nehmen, kann jedoch mehr zur Neuorientierung und Effizienzsteigerung von Vorbeugung und Heilung beitragen als die Fortschreibung konventioneller Forschungsprogramme.

Schlüsselwörter: Kontrollierter Konsum - Opiate - Kokain/ Crack

Addiction therapy and the controlled consumption of opiates/heroin and cocaine/crack: Quite naturally addiction sciences are more interested in the causes and treatments of addiction than in the conditions and extent of a possible controlled use of addictive substances. This article argues that a closer look at extent and quality of controlled consumption can produce useful insights for therapeutic and more general (deontic) responses to the drug problem, e.g. drug legislation.

Key words: Controlled Consumption - Opiates - Cocaine/ Crack

\section{Einleitung}

In der Öffentlichkeit, teilweise auch in der Wissenschaft, unterscheidet man streng zwischen Genussmitteln wie Kaffee, Tee, Zigaretten und Alkoholika einerseits und Rauschmitteln (gelegentlich auch Rauschgifte, Suchtgifte, Betäu-

Suchttherapie Sonderheft 2001; 2: S28-S31

(c) Georg Thieme Verlag Stuttgart · New York

ISSN 1439-9903

\section{Sebastian Scheerer}

Institut für Kriminologische Sozialforschung, Universität Hamburg bungsmittel oder Drogen genannt) wie Heroin, Haschisch, LSD, Ecstasy und Kokain andererseits. Was die beiden Kategorien unterscheidet, ist nicht das Risiko der Gesundheitsschädigung: Dass das Rauchen von Zigaretten und das Trinken von Alkohol ganz erhebliche Risiken birgt, die sich mit denen des Konsums mancher verbotener Drogen durchaus messen können, steht heute wohl außer Frage. Wenn es gerechtfertigt sein sollte, zwischen Genussmitteln und Rauschmitteln dennoch einen kategorialen Unterschied anzunehmen, dann kann diese Rechtfertigung nach aktueller Sicht nicht mehr darin liegen, dass die eine Gruppe von Drogen „harmlos“ ist, die andere aber „gefährlich“, sondern nur in der quantitativen Verschiedenheit des Risikos: Genussmittel werden typischerweise vernünftig und kontrolliert konsumiert und nur eine Minderheit verfällt der Sucht - Suchtmittel hingegen werden typischerweise aus Neugierde probiert, erweisen sich aber in der Regel bald als stärker als der Wille des einzelnen Menschen und führen typischerweise immer dann zu Sucht und Tod, wenn nicht rechtzeitig die richtige Hilfe von außen kommt. Selbst diejenigen, die eine sehr kritische Sicht der Drogenpolitik und der Suchtmedizin pflegen und z.B. für die Entkriminalisierung von Cannabisprodukten plädieren, greifen diese grundsätzliche Unterscheidung nicht an, sondern fordern lediglich deren sachgerechte Durchführung: Wenn alles darauf hindeutet, so die Legalisierer, dass Cannabis ähnlich wie andere Drogen, die wir als Genussmittel zugänglich machen, von der großen Mehrheit der Konsument(inn)en ohne Kontrollverlust gebraucht werden kann, dann ist es nicht sachgerecht, diese Substanz mit demselben Nachdruck zu verbieten und strafrechtlich zu verfolgen wie die wahren Suchtdrogen, also etwa Heroin und Kokain.

Die Unterscheidung der Genussmittel von den Betäubungsmitteln gründet auf dieser Überzeugung: Während all diese Substanzen Risiken bergen, können die Menschen die Genussmittel im Normalfall beherrschen - während sie im Normalfall früher oder später den Kampf gegen die Rauschmittel verlieren und von ihnen beherrscht werden. Bei Genussmitteln ist der Kontrollverlust die Ausnahme, bei Rauschgiften die Regel. Alkohol und Tabak produzieren zwar enorme Kosten und Probleme, aber die Freigabe der Rauschgifte wäre eine Katastrophe. Letztere nämlich tendieren dazu, Gesellschaften zu überschwemmen und unter sich zu begraben, wenn man sie nicht mit allen Mitteln zurückhält. Die Beratende Opium-Kommission des Völkerbundes hatte dies so illustriert: Wie „Wasser unter ungeheurem Druck“ versuche das Rauschgift, sich einen Weg zu bahnen und „mit unwiderstehlicher Gewalt auf die entferntesten Stellen des 
Systems ... zu verteilen; ohne Unterlass und gleichsam automatisch drängt es danach, jeden Fehler, jede schwache Stelle des eisernen Walles, der es umgibt, ausfindig zu machen; es sucht nach jedem Mittel, diesen Wall zu durchbrechen und bemüht sich unablässig, seine Bande abzuschütteln ...."1 - aus dieser Zeit stammt auch das belief system der Medizin, dass man zwar Alkohol gelegentlich zu sich nehmen und Tabakerzeugnisse gelegentlich rauchen, nicht aber Heroin gelegentlich zu sich nehmen könne, ohne süchtig zu werden. ${ }^{2}$ Regelmäßiger Opiatkonsum führe unweigerlich zu Sucht, charakterlicher Entkernung, Verflachung der Gesinnung, krankhaften Organveränderungen und frühem Tod [3].

Die Theorie der Drogenwirkung diktierte auch die Theorie der Ursachen des Drogenkonsums. Wenn Drogenkonsum zu Sucht und Tod führt, dann kann der Grund für den Griff zur Droge nur ein verborgener Todeswunsch sein: der Wunsch, sich zu zerstören, zumindest einer Realität zu entfliehen, an der man gescheitert war - aus Krankheit, Langeweile oder Charakterschwäche, als Opfer von Ausbeutung oder Unterdrückung, Depression oder Frustration. ${ }^{3}$

Dass manche Persönlichkeiten trotz Opiat- und Kokainkonsums erhebliche professionelle Leistungen zu erbringen und ein hohes Lebensalter zu erreichen vermochten, galt lange Zeit als anekdotisch interessantes, aber auf Einzelfälle begrenztes und in wissenschaftlicher Hinsicht eher irrelevantes oder gar ablenkendes und verharmlosendes Wissen. Erst um 1980 herum machen sich einzelne Wissenschaftler mit ihren Forschungen und mit ihrer These bemerkbar, dass es sich beim kontrollierten Konsum durchaus um ein quantitativ erhebliches und qualitativ bemerkenswertes Phänomen handele, das geeignet sei, konventionelle Überzeugungen infrage zu stellen und wichtiges neues Wissen zu generieren.

Seither sind zwei Jahrzehnte ins Land gegangen. Zeit genug, um eine Zwischenbilanz zu ziehen. Handelt es sich beim Phänomen des kontrollierten Konsums gefährlicher Suchtdrogen eher um eine Randerscheinung oder um eine soziale Tatsache mit Implikationen auch für die Suchtmedizin?

\footnotetext{
1 Zitiert nach [1]; Wie diese verdinglichende und dämonisierende Sichtweise, die den Substanzen selbst eine übermenschliche Kraft zuschreibt, noch heute gelegentlich weiterwirkt, zeigt sich bei genauerer Betrachtung bekannter Buchtitel, in denen von der „Weltmacht Droge“ die Rede ist oder von so gewohnten Slogans wie „Keine Macht den Drogen“ ...

2 Der Aussage: „Du kannst Heroin gelegentlich nehmen, ohne jemals süchtig zu werden“ widersprachen Anfang der 70er Jahre 90\% der Amerikaner; vgl. [2] (S. 1218: „... Literatur zeigt, dass sich buchstäblich Hunderte von Studien mit dem einen oder anderen Aspekt der Abhängigkeit befassen, jedoch nur weniger als ein Dutzend in der Hauptsache von gelegentlichem Heroingenuss handeln. - Selbst als die Existenz nichtabhängigen Heroingebrauchs anerkannt war, wurde seine Bedeutung durch die Feststellung heruntergespielt, dass es sich nur um ein kurzes Übergangsstadium zwischen experimentellem Genuss und Abhängigkeit handele ...").

3 Vgl. [4, 5]; da nicht alle "Rauschgifte“ berauschen und auch nicht alle „Betäubungsmittel“ betäuben, ihre Gemeinsamkeit vielmehr in Sucht und Zerstörung bestehe, solle man sie besser "Suchtgifte“ nennen, meinte denn auch Otto Zekert [6].
}

\section{Quantität und Qualität kontrollierten Konsums}

Dass sich nicht alle von der Prohibition erfassten Drogen letztlich als stärker erweisen als diejenigen, die sie konsumieren, lässt sich ganz einfach am Beispiel des Cannabiskonsums zeigen [7]. Die herkömmliche Vorstellung eines pharmakologisch determinierten Suchtgeschehens scheitert aber auch an der Frage, wie es denn kommen kann, dass manche IntensivKonsumenten von Heroin [8,9] und Kokain ${ }^{4}$ [10-12] süchtig werden, andere aber nicht.

Offenbar ist es die Droge nicht allein, sondern nur in relativierender Wechselwirkung mit set and setting (Norman Zinberg), die das Spektrum dessen erklären kann, was bei umfassender und differenzierender Betrachtung nach dem Drogenkonsum in kurz-, mittel- und langfristiger Hinsicht passiert. Entscheidend für die Frage, ob jemand süchtig wird oder nicht, scheint danach nicht der Drogenkonsum als solcher, sondern vielmehr die Art und Weise des Konsums, insbesondere die Befolgung von Regeln und Ritualen (rules and rituals nach Wayne Harding).

Die bisher ungelöste Frage, warum innerhalb einer einzigen Konsumentengruppe mit identischen Regeln und Ritualen manche Gruppenmitglieder ihren Konsum zu kontrollieren vermögen, andere aber nicht, beantwortet Grund [17] mit seinem Feedback Model of Drug Use Self-Regulation und der DARRLS-Trias als dessen Kernstück (Drug Availability, Rituals and Rules, Life Structure). ${ }^{5}$ Danach ist es vor allem denjenigen Konsument(inn)en möglich, trotz Drogenkonsums das Abgleiten in die Sucht zu vermeiden, die keine Versorgungsengpässe kennen und ihre Energie auf die Bewältigung anderer

\footnotetext{
${ }^{4}$ Unter den illegalen Drogen kommt in den USA Kokain und Crack noch das höchste Missbrauchspotenzial zu, die eine Missbrauchsrate von 10-25\% aufweisen. Praktisch werden alle anderen Drogen von $90 \%$ der Konsumenten nicht miss-, sondern gebraucht. Vgl. [13-16].

5 "Although this feedback model is circular, it is not a closed and independent circuit. The three cornerstones of the feedback model are each the result of distinctive variables and processes. Drug availability is determined by price, purity and accessibility, which are mediated by market factors and governmental regulations ... Rituals and rules are the product of culturally defined social learning processes ... The shape and degree of life structure are the product of the regular activities, relationships and ambitions which may be drug related or not. General socio-economic factors and actual living conditions, personality structure and the prevalence of (non drug related) psycho-social problems, and cultural factors may further determine life structure ... Clearly, external stimuli can impact on the feedback system, in particular on its ability to support controlled and adjust uncontrolled use ... The social definition of drugs and their users, embodied in drug policy, can be seen to affect the model at all three cornerstones. - Rituals and rules determine and constrain the patterns of drug use, preventing an erosion of life structure. A high degree of life structure enables the user to maintain a stable drug availability, which is essential for the formation and maintenance of efficient rules and rituals. Self-regulation of drug consumption and its (unintended) effects is thus a matter of a precarious) balance of a circularly reinforcement chain ..... Vgl. jüngst auch [18]. Decortes Ergebnisse von Tiefeninterviews mit 111 erfahrenen Kokaingebrauchern in Antwerpen bestätigen Ergebnisse von Untersuchungen in Amsterdam, Rotterdam, Barcelona, Frankfurt a.M., Turin, San Francisco, Toronto, Schottland und Australien.
} 
Alltagsprobleme persönlicher, sozialer und beruflicher Art konzentrieren, ihren Alltag also einigermaßen befriedigend strukturieren können.

Kann es sein, dass die auf Drogenverknappung zielende Drogenpolitik gerade das Gegenteil dessen bewirkt, was sie beabsichtigt? Je stärker der Verfolgungsdruck, desto geringer die Chancen der Konsument(inn)en, sich zu informellen Gruppen zusammenzufinden und gewisse Gewohnheiten und Regeln zu entwickeln, die den Drogenkonsum domestizieren und in einen nicht nur von Drogen dominierten Alltagsrhythmus einbinden. Warum spielen derartige Aspekte in der herrschenden Einstellung zur Droge und in der seit langem praktizierten Drogenpolitik keine Rolle? Die Antwort liegt auf der Hand. Das Axiom der kategorialen (und rechtlichen) Trennung der psychoaktiven Substanzen in Genussdrogen einerseits und Betäubungsmittel andererseits ist ja gerade die prinzipielle Unbeherrschbarkeit der zur letztgenannten Kategorie gehörenden Drogen. Da müsste es paradox erscheinen, die Politik von der Suche nach kontrollbegünstigenden Bedingungen bestimmen zu lassen. Die Nichtbeachtung des kontrollierten Konsums führt nach Art einer self-fulfilling prophecy als mit-entscheidender Faktor dazu, dass die Bedingungen der Möglichkeit kontrollierten Konsums häufig gerade dort, wo sie ohne die Repression entstehen und viele Konsument(inn)en vor der Sucht bewahren könnten, sich nicht entwickeln.

\section{Diskussion}

Die Erforschung des kontrollierten Konsums von so genannten Suchtdrogen kann vor falschen Vorstellungen einer zwangsläufigen Suchtentwicklung bewahren und insofern eine notwendige und berechtigte Enttabuisierung fördern. Die verbreitete Befürchtung einer verharmlosenden Wirkung der Informationen über kontrollierten Konsum dürfte gleichwohl nicht berechtigt sein, handelt es sich ja nicht um eine fälschliche Negierung von Risiken, sondern um eine realistische Komplettierung des bislang durch Halbwahrheiten und deterministische Kausalmodelle dominierten Drogendiskurses.

Das positive Potenzial der Erkundung der Verbreitung und der Bedingungen kontrollierten Konsums liegt in der Bereicherung der Optionen einer künftigen Suchtpolitik und -medizin. Das Paradigma der akzeptierenden Drogenarbeit wird gestärkt, die bipolare rechtliche Rahmung des gesellschaftlichen Umgangs mit psychoaktiven Substanzen mit ihrer strikten Ungleichbehandlung von einigen Drogen als Genussmitteln einerseits und anderen als Rauschgiften andererseits wird herausgefordert und der Weg zur wissenschaftlichen Grundlegung einer neuen Drogenpolitik und Suchtmedizin wird geebnet. Denn wenn es „normale“ Menschen sind, die aus „normalen“ Motiven der Freizeitgestaltung zu Drogen greifen, dann verantworten auch nicht die Dealer, sondern die Konsumenten die Konsumentscheidung. Die große Mehrheit der Drogenkonsumenten hat sich von Lebensplänen, Karrieremustern und dem gesellschaftlichen und finanziellen Weiterkommen keineswegs verabschiedet. Wenn sie Drogen nehmen, versinken sie nicht in ihrer eigenen Höhle, sondern feiern mit anderen, was es zu feiern gibt: das Ende einer anstrengenden, aber erfolgreichen Arbeitswoche, die Möglichkeit, unter Freunden und Bekannten aus sich herauszugehen, neue und interessante Erfahrungen zu gewinnen. Man zieht sich nicht zurück, sondern verfolgt die alten legitimen Ziele mit neuen, innovativen Mitteln. ${ }^{6}$

Zur Zeit der Entstehung der Drogengesetzgebung mag das anders gewesen sein. Um aber den Drogenkonsum in der Gegenwartsgesellschaft zu erklären, nützen pathologisierende Erklärungen nichts mehr. Der aktuelle Drogenkonsum ist integrierter Bestandteil einer in ihren Werten und Lebensstilen gewandelten Gesellschaft, in der traditionelle Werte (Gehorsam, Disziplin usw.) durch die Aufwertung „postmoderner" Ideale wie Selbsterfahrung und -entfaltung, Kreativität und Gemeinsinn stark relativiert wurden. In diesem Zusammenhang erfuhren auch „Spannung“, „Abenteuer“, „Aufregung“, „Unterhaltung“ und „Erlebnisse“ - Gefühlszustände, für die man auch Drogen einsetzen kann - eine Aufwertung. Die „postmoderne“ Erlebnisgesellschaft fördert die intelligente Nutzung der Warenwelt zu subjektiven Erlebniszwecken: vom Abenteuertourismus bis zu Snowboardfahren und Tiefseetauchen. Wenn aber Drogen bei gleichem oder geringerem Risiko mindestens ebenso viel excitement, pleasure und entertainment ${ }^{7}[20,21]$ versprechen, muss es verlogen wirken, wenn die Gesellschaft dem mutigen Snowboarder zwar riskanteste Schussfahrten erlaubt, ihm aber bei Androhung von Kriminalstrafe verbietet, die Freunde beim Après-Ski nicht nur mit Alkoholika, sondern auch mit einer kleinen Prise Kokains zu erfreuen? ${ }^{8}$

\footnotetext{
${ }^{6}$ So zeigt etwa eine 1996 in Norddeutschland durchgeführte Explorationsstudie [19], dass knapp 40\% der in Diskotheken, Raver-Clubs und ähnlichen Orten befragten 669 Personen angeben, Ecstasy zu konsumieren, jedoch von diesen nur 9\% häufiger als einmal wöchentlich, hingegen 56\% seltener als wöchentlich. Befragt nach ihren Konsummotiven und subjektiven Drogenerwartungen gaben rd. $54 \%$ an, es gehe ihnen vorrangig um ein „gutes Feeling“, knapp 30\% sagten, sie könnten mittels Designerdrogen besser „durchtanzen/durchmachen“; für gut 19\% stehen „Harmonie/Spaß/Feiern mit anderen im Vordergrund“. Demgegenüber begründen die NichtKonsumenten ihren Konsumverzicht zu gut zwei Drittel mit befürchteten „unerwünschten Nebenwirkungen“ (26,0\%) bzw. „Schädigungen/Gefahren“ (41,8\%).

7 So ist z.B. der Kokainkonsum im „bürgerlichen Milieu“ keineswegs ein Protestsymbol; er dient auch nicht der Bewusstseinserweiterung oder der unbewussten Selbstmedikation; er ist integrierter Bestandteil der Konsumkultur, weil auch die Drogenkonsumenten vor allem einfach Spaß, Unterhaltung, Erlebnisse suchen und der Drogenkonsum der Gebrauch einer Ware zur Steigerung des Lebensgenusses ist. Vgl. [22]: „... es ist alles unheimlich grenzenlos möglich.“ [23].

8 Riskant ist der Drogengebrauch nicht zuletzt künstlich - nämlich deswegen, weil er verboten ist. Die Drogen unterliegen keiner Lebensmittelkontrolle. Mit einer Legalisierung würde die Kontrolle über Qualität und Wirkstoffkonzentrat überhaupt erst einsetzen. Auch werden die prohibitionsbedingten Extra-Gefahren, die sich aus der Notwendigkeit des Schmuggels und der Vermeidung von Entdeckung ergeben und die sich auf die Konzentration des Wirkstoffs ebenso risikoerhöhend auswirken wie auf die sozialen und hygienischen Bedingungen des Konsums und die Möglichkeiten einer rituellen Risikominderung, einer Verlagerung des Konsums von hochkonzentrierten Darreichungsformen auf wirkstoffärmere Angebote weichen. Zu einer Drogenkultur, die den Namen verdient, vgl. [24]; diese und weitere Aufsätze finden sich auch in [25].
} 
Wenn die neueren Ergebnisse zur Verbreitung und zu den Bedingungen kontrollierten Konsums von so genannten Suchtdrogen durch weitere Forschungen belegt werden, wird sich die Suchtmedizin mit größerer therapeutischer Freiheit auch denen widmen können, deren Behandlung heute noch vom Drogenrecht enge Grenzen gesetzt sind. Denn dann ist die bipolare Drogengesetzgebung nicht länger zu begründen. Dann unterscheiden sich die heute verbotenen Drogen nicht wesentlich von anderen Objekten, mit denen Menschen zur Bewältigung oder Transzendierung des Alltags und zur Heilung von Krankheiten umgehen [26]. - Eine künftige Drogenpolitik würde wohl die Legitimität der Nachfrage nach psychoaktiven Substanzen anerkennen, was nicht ohne Folgen für die Therapie-Palette bliebe, die sich wohl zahlreicher repressiver Elemente entledigen und der Angebotsstruktur etwa der heutigen Raucher-Entzugs- und Entwöhnungstherapien angleichen könnte. Überhaupt würde die strikte Trennung von Genuss- und Rauschmitteln wohl kaum noch zu begründen sein. Die internationalen Abkommen, die eine grundlegende Reform auf der Basis neuer wissenschaftlicher Erkenntnisse durch die Festschreibung veralteter Vorstellungen verhindern, werden sich nicht ewig gegen den wissenschaftlichen Fortschritt stemmen können. Auch sind sie alle durch Schreiben an den UN-Generalsekretär kündbar: Kündigungstermin für die Single Convention ist der 30. Juni mit Wirkung zum Jahresende; das Wiener Abkommen von 1988 kann jederzeit gekündigt werden (mit Wirkung nach Ablauf eines Jahres, beginnend mit dem Eingang der Kündigungserklärung).

\section{Literatur}

${ }^{1}$ Völkerbund und Rauschgiftbekämpfung. In: Informationsabteilung des Völkerbundes (Hrsg). Genf (ohne Jahresangabe): Druck der Vernay A.-G., Wien, IX., Canisiusgasse 8-10, (1934); 2

${ }^{2}$ Harding W. Kontrollierter Heroingenuss - ein Widerspruch aus der Subkultur gegenüber herkömmlichem kulturellem Denken. In: Völger/Welck (Hrsg). Rausch und Realität. 3 Bde. Reinbek: Rowohlt, 1982; (Bd. 3): 1217-1231

${ }^{3}$ Kielholz P, Ladewig D. Die Abhängigkeit von Drogen. Dt. Taschenbuch-Verlag. München, 1973: $39 \mathrm{f}$.

${ }^{4}$ Merton RK. Social Structure and Anomie. In: Social Theory and Social Structure. rev. ed (Hrsg). Glenwe, III. The Free Press. New York, 1957: 131-194

${ }^{5}$ Merton RK. Continuities in the Theory of Social Structure and Anomie. Merton RK. Social Theory and Social Structure. rev. ed. New York, 1957: 131-194

${ }^{6}$ Zekert O. Opiologie, ein Beitrag zur Geschichte des Opiums und seiner Wirkstoffe HMW-Jahrbuch 1957. Wien: Heilmittelwerke, 1956; 147

${ }^{7}$ Zimmer L, Morgan JP. Marijuana Myths, Marijuana Facts. A Review of the scientific evidence. New York, San Francisco: The Lindesmith Center, 1997: 27-30

${ }^{8}$ Harding, a.a.O. Kontrollierter Heroingenuss - ein Widerspruch aus der Subkultur gegenüber herkömmlichem kulturellem Denken. In: Völger/Welck (Hrsg). Rausch und Realität. 3 Bde. Reinbek: Rowohlt, 1982; (Bd. 3): 1217-1231

${ }^{9}$ Hess H, Behr R, Klös P. Projektgruppe KiF

${ }^{10}$ Waldorf D, Reinarman C, Murphy S. Cocaine Changes. The Experience of Using and Quitting. Philadelphia: Temple University Press, 1991
${ }^{11}$ Cohen PDA. Crack in the Netherlands: Effective Social Policy is Effective Drug Policy. In: Reinarman C, Levine HG (Hrsg). Crack in America. Berkeley, Los Angeles, London: University of California Press, 1997: 214-224

${ }^{12}$ Hess H, Behr R, Klös P. Projektgruppe KiF

${ }^{13}$ Lap M, Polak F. Gründe für eine Legalisierung: Das Gesundheitsargument. In: Nolte F, Quensel S, Schulze A (Hrsg). Wider besseres Wissen. Die Scheinheiligkeit der Drogenpolitik. Bremen: Temmen, 1996: 206-218

${ }^{14}$ Nicholson T. The Primary Prevention of Illicit Drug Problems. The Journal of Primary Prevention 1992; 14: 275-288

${ }^{15}$ Cohen P, Sas A. Ten years of Cocaine.: University of Amsterdam, 1993

${ }^{16}$ Cohen P, Sas A. Ten years of Cocaine. Nachuntersuchung: University of Amsterdam, 1994

${ }^{17}$ Grund JP. Drug Use as a Social Ritual: Functionality, Symbolism and Determinants of Self-Regulation. Instituut voor Verslavingsonderzoek. Rotterdam, 1993; Kapitel 17 (zit.n. Internet, ohne Seitenangabe)

${ }^{18}$ Decorte T. The Tamine of Cocaine: Cocaine Use in European and American Cities. Amsterdam: Vu Univ Pr, 2001

${ }^{19}$ Wiklens W et al. Illegal oder (l)egal - Rechtlicher Status und Konsum von MBDB und anderen Ecstasy-Wirkstoffen. In: Wilkens W (Hrsg). Hamburg eine Szenebefragung aus dem Herbst 1996: Hamburger Institut Drogen und AIDS, 1997

20 O'Malley P, Mugford S. The Demand for Intoxicating Commodities: Implications for the „War on Drugs“. Social Justice 1991; 18: 49-75

${ }^{21}$ Campbell C. The Romantic Ethic and the Spirit of Modern Consumerism. Oxford: Blackwell, 1987

${ }^{22}$ Hess H, Behr R, Klös P. Projektgruppe KiF

${ }^{23}$ nnnbitte Angabe des Autors ergänzennnn. Kokain in Frankfurt Konsummuster und Verteilerhandel im bürgerlichen Milieu. Forschung Frankfurt 1999; 4: 30-37

${ }^{24}$ Marzahn C. Plädoyer für eine gemeine Drogenkultur. In: Beck J et al (Hrsg). Das Recht auf Ungezogenheit. Reinbek: Rowohlt, 1983: 105-134

${ }^{25}$ Marzahn C, Tibi B. Über Genuss und Geist. Bremen: Temmen, 1994

${ }^{26}$ Schmidt-Semisch H. Drogen als Genussmittel. München: AG SPAK, 1992

Prof. Dr. Sebastian Scheerer

Institut für Kriminologische Sozialforschung

Universität Hamburg

Troplowitzstraße 7

22529 Hamburg

E-mail: scheerer@uni-hamburg.de 